\title{
DISTÚRBIO ONDULATÓRIO DE LESTE COMO CONDICIONANTE A EVENTOS EXTREMOS DE PRECIPITAÇÃO EM PERNAMBUCO
}

\author{
MACHADO, Célia Cristina Clemente - celiamachado.machado@gmail.com \\ NÓBREGA, Ranyére Silva \\ OLIVEIRA, Tiago Henrique de \\ ALVES, Keyla Manuela Alencar da Silva \\ Programa de Pós-graduação em Geografia (PPGEO) / \\ UFPE - Universidade Federal de Pernambuco
}

\begin{abstract}
RESUMO
Os Distúrbios Ondulatórios de leste (DOLs) são sistemas de escala sinótica, de longitudes horizontais da ordem de $1.000 \mathrm{Km}$ ou mais, que ocorrem na baixa Troposfera Tropical. Despertaram o interesse de vários pesquisadores durante anos devido a sua influência na precipitação em regiões tropicais. $O$ sistema DOL produz uma forte liberação de calor latente ocasionando perturbações nos campos de vento e pressão, assim modificando as condições de tempo durante sua trajetória. Deste modo, o objetivo deste artigo é analisar a influência dos DOL como condicionantes para intensificação de eventos de precipitação em Pernambuco, analisando o evento ocorrido em junho de 2010 e os impactos sobre o município de Palmares. A ocorrência de um DOL acoplado a condições de temperaturas de superfície do mar anômalas modulou a intensificação de um sistema convectivo de mesoescala, que produziu precipitações acima dos padrões normais durante 3 dias. O sistema de DOL pelo seu tempo de duração e pelos valores de precipitação superiores a $100 \mathrm{~mm}$ indicam que esse distúrbio é um sistema importante para ocorrência de chuva na costa leste do NEB. Quanto aos impactos ocorridos no município de Palmares, uma série de fatores foram responsáveis e não apenas o evento em si. As elevadas precipitações ocorridas nas semanas anteriores ao evento e sua concentração em três dias principalmente nas cabeceiras, as características do solo e do relevo e a ocupação das planícies de inundação pela população agravaram para proporções catastróficas um evento já em si muito intenso.
\end{abstract}

PALAVRAS CHAVES: Cheia, evento extremo, precipitação, Goes-12, Palmares.

DISTURBANCE OF EAST WAVE AS CONDITIONING THE EXTREME PRECIPITATION EVENTS IN PERNAMBUCO

\section{ABSTRACT}

The Easterly Waves (DOL) are synoptic scale systems that occur in the lower Tropical Troposphere, with horizontal longitudes of about $1.000 \mathrm{~km}$ or more, that occur in the low Tropical Troposphere. It has aroused several researchers' interest during years due to their influence in the precipitation of tropical regions. DOL system produces a strong liberation of latent heat provoking disturbances in wind and pressure fields, changing weather conditions during its trajectory. Therefore, this article aims to analyze DOL influence as a conditioner to the intensification of precipitation events in Pernambuco state, by analyzing the event that occurred in June 2010 and its impacts over Palmares municipality. The occurrence of a DOL coupled to anomalous sea surface temperatures pitched the intensification of a Mesoscale Convective System (MCC), that provoked precipitations above the normal standards for three days. Dol systems due to their duration time and to their precipitation value over $100 \mathrm{~mm}$, indicate that it is an important system to rain occurrence in Brazilian Northeast. Concerning the impacts that occurred in Palmares municipality, a series of factors were responsible e not just the event by itself. The previous weeks heavy rains and its concentration during three days mainly in the headwaters, the soil and relief characteristics and the human occupation of the flooding plains increased to catastrophic proportions an event already very intense.

KEYWORDS: Flood, extreme event, precipitation, Goes-12, Palmares.

\section{Resumen}

Los Disturbios Ondulatorios del Este (DOLS) son sistemas de escala sinóptica, de longitudes horizontales del orden de $1.000 \mathrm{~km}$ o más, que ocurren en la baja tropósfera tropical. Esto despertó el interés de muchos investigadores durante años, debido a su influencia en las precipitaciones de las regiones tropicales. El sistema DOL, produce una fuerte liberación de calor latente provocando perturbaciones en los campos de presión y viento, produciendo cambios en las condiciones meteorológicas durante su trayectoria. En este sentido, el objetivo de este trabajo es analizar la influencia del sistema DOL como condicionante para la intensificación de las lluvias en Pernambuco, analizando el evento ocurrido en junio de 2010 y el impacto en la ciudad de Palmares. Ya que la ocurrencia de una perturbación de DOL acoplado a condiciones de temperatura de superficie del mar anómala, moduló la intensificación de un sistema convectivo de mesoescala, que produjo la precipitación por encima del rango normal durante 3 días. El sistema DOL por su duración y las 
cantidades de precipitaciones superiores a $100 \mathrm{~mm}$ indican que este disturbio es un sistema importante para la ocurrencia de lluvias en la costa este de la Región Nordeste de Brasil. En cuanto a los impactos ocurridos en la ciudad de Palmares, una serie de factores fueron los responsables, no sólo el evento mismo, las fuertes lluvias que se produjeron en las semanas previas al evento y su concentración en tres días, sobre todo en las cabeceras de los ríos, además de las características del suelo y geomorfología, y finalmente la ocupación de las planicies de inundación por la pueblo, tuvieron efectos exponenciales en las proporciones catastróficas de un evento clasificado como muy intenso.

PALABRAS CLAVE: Inundación, evento extremo, precipitación, Goes-12, Palmares

\section{Résumé}

Les Perturbations Ondulatoires d'Est (DOLs) se présentent comme des systèmes d'échelle synoptique, avec des longitude horizontal de $1000 \mathrm{~km}$ ou plus, qui se produisent dans la Basse Troposphère Tropicale. Elles ont suscité l'intérêt de plusieurs chercheurs pendant des années en raison de son influence sur les précipitations dans les régions tropicales. Um DOL produit une forte libération de chaleur latente ocasionant des pérturbations de préssion et de vent, modifiant de cette forme, les conditions du temps pendant son trajectoire. Ainsi, l'objectif de cet article est d' analyser l'influence du DOL comme un conditionnant pour l'intensification des événement qui ont eu lieu en Juin 2010, et ses répercussions sur la commune de Palmares. La manifestation d'un DOL liée a des températures de surface anormal a provoqué l'intensification d'un complexe convectif de mésoéchelle (CCM) qui a produit des précipitations supérieure à l'échelle pendant trois jours. Le temps de duration e les valeurs supérieure a $100 \mathrm{~mm}$ des DOL indiquent que cette perturbation est un important systèm pour la maniféstation de pluie dans le Nord-Est Brésilien. En relation aux répercussions sur la commune de Palmares, un certain nombre de facteurs ont été résponsables et non seulement l'événement lui-même. Les fortes précipitations qui ont eu lieu quelques semaines avant l'événement et sa concentration pendant trois jour, principalment sur les eaux de source, les caractéristiques du sol et du relief et l'occupation humaines des plaines d'inondation ont aggravé le cas dans des proportions extrêmes, un événement en soi très intense.

MOTS-CLÉS: Inondation, événement extrême, précipitations, GOES-12, Palmares.

\section{Introdução}

Os Distúrbios Ondulatórios de Leste (DOLs) são sistemas de escala sinótica que ocorrem na baixa Troposfera Tropical, e quando se formam na região do Oceano Atlântico Sul apresentam deslocamentos para o oeste intensificando-se à medida que se aproximam da costa leste da Região Nordeste do Brasil (CHOU, 1990; SILVA, 2010). Estes sistemas oscilam com periodicidade variando entre 3 a 9 dias e velocidade de propagação entre 10 a $15 \mathrm{~m} / \mathrm{s}$ (MEKONNEN et al., 2006; ALVES et al., 2008).

Silva (2010) e Cavalcanti et al. (2009) descrevem que pesquisas sobre DOLs realizadas procuraram analisar as interações de ondas de leste com a orografia das montanhas da America Central e México e com o anticiclone da região, como as descritas em Zehnder et al. (1999). Os autores concluíram que a interação dos DOLs com o relevo resultou em um aumento da vorticidade relativa a sotavento do terreno, o que pode ser aplicado nas fases iniciais do desenvolvimento de instabilidade convectivas.

Coutinho \& Fisch (2007) e Barbosa (2005) corroboram afirmando que um DOL pode intensificar os sistemas meteorológicos de perturbação convectiva, tais como as linhas de instabilidades que se propagam para o interior da Amazônia ou ainda associar-se com a ocorrência de tempestades tropicais ou mesmo furacões na parte norte do Atlântico Equatorial (COOK et al., 2004).

Para Mota (1997), o sistema DOL modifica as condições de tempo ao longo de sua trajetória e a sua principal fonte de energia parece ser a liberação de calor latente através de processos de convecção profunda. Deste modo, os DOLs 
ocasionam perturbações nos campos de vento e de pressão e apresentam deslocamento característico de leste para oeste, no mesmo sentido dos ventos alísios.

Barbosa (2005) afirma que existe ampla evidência observacional de que os DOLs atuam na costa Norte-Nordeste (N-NE) do Brasil durante quase todo o ano enquanto que Machado et al. (1996) através da realização de estudo sobre as flutuações de Radiação de Onda Longa Emergente (ROLE) sobre a América do Sul obteve resultados demonstrando que a variabilidade ao longo do dia apresenta contribuição significativa dos DOLs.

Deste modo, o objetivo deste artigo é analisar a influência do Distúrbio Ondulatório de Leste como condicionante para intensificação de eventos de precipitação em Pernambuco, analisando o evento ocorrido em junho de 2010 e os impactos sobre o município de Palmares.

\section{Materiais e métodos}

\section{1. Área de estudo}

Para esta análise foi selecionado o município de Palmares, inserido totalmente na bacia hidrográfica do rio Una (Figura 1), localizado na latitude de -08041'00" e longitude de -35035'30", com altitude de 125 metros. A escolha deve-se a dimensão do impacto durante o evento extremo de precipitação de 2010 (GIRÃO et al., 2012) e também a disponibilidade de dados necessários para o estudo.

O município possui clima predominante do tipo quente e úmido, com chuvas de inverno e temperatura máxima de $32^{\circ} \mathrm{C}$ e mínima de $18^{\circ} \mathrm{C}$. A sua topografia é predominantemente ondulada e caracteriza-se por um conjunto de morros e colinas com altitudes não superiores a $120 \mathrm{~m}$ e pediplanos resultantes do alargamento do vale do rio Una e seus afluentes.

O rio Una nasce na cidade de Capoeiras, agreste de Pernambuco, a uma altitude de 900 metros e percorre aproximadamente $255 \mathrm{~km}$ até o Oceano Atlântico. A sua bacia está localizada no limite sul do litoral de Pernambuco, entre - 8017'14" S e - $8^{\circ} 55^{\prime} 28^{\prime \prime}$ de latitude e - 35007'48" e - 36042'10" de longitude (Figura 1) e apresenta uma área total de $6274 \mathrm{~km}^{2}$.

O rio corta o município de Palmares na direção oeste-leste até a Fazenda Couceiro, onde toma a direção sul até encontrar a sede do município, tomando novamente a direção leste. O mesmo corriqueiramente apresenta anualmente algumas enchentes no período de maior precipitação atingindo alguns municípios como Palmares, Catende, Água Preta e Barreiros. 


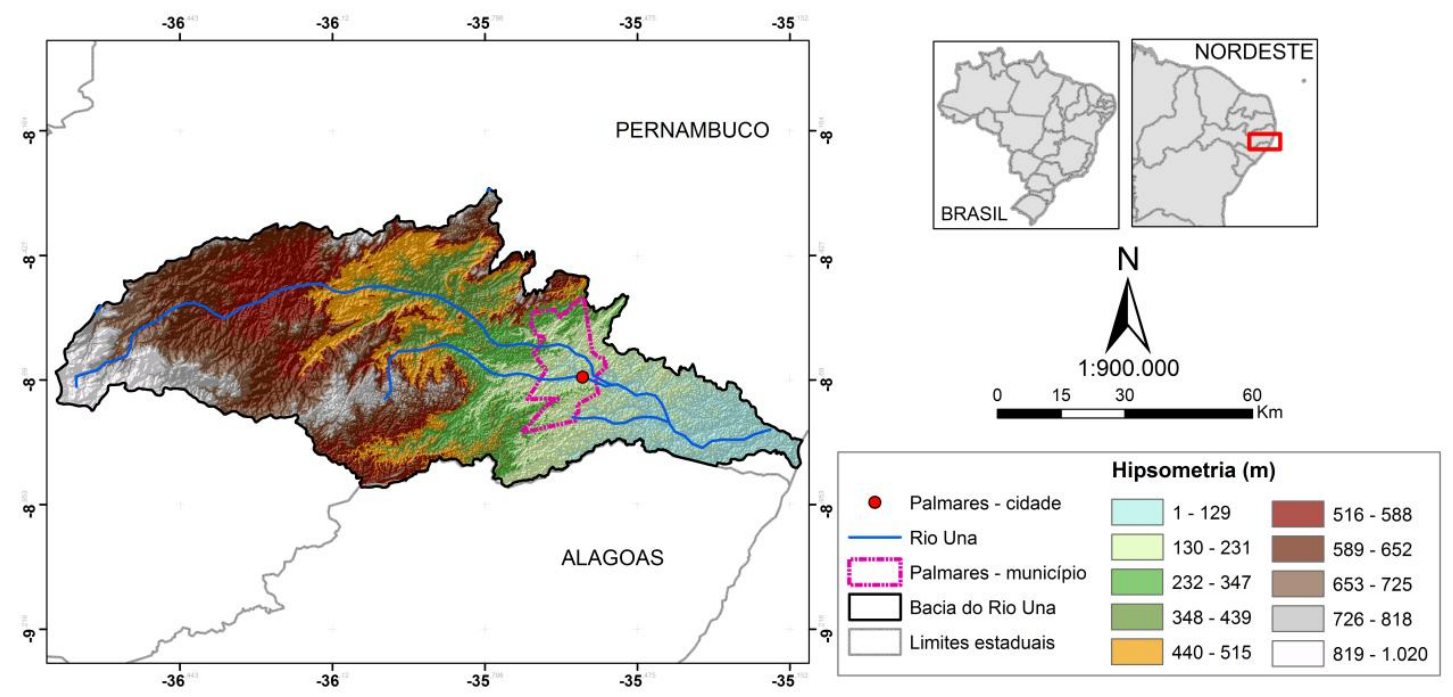

Figura 1 - Localização e do município de Palmares inserido na bacia hidrográfica do rio Una e representação da hipsometria da bacia.

\subsection{Metodologia}

Para a análise sobre a gênese, deslocamento, e dimensão espacial do sistema convectivo foram utilizadas imagens do canal infravermelho do satélite GOES12 dos dias 17 e 18 de junho de 2010, com resolução espacial de 4 km e resolução temporal de 15 minutos, disponíveis on-line pelo site da Administração Aeronáutica e Espacial Nacional (NASA), Centro de Vôo Espacial Goddard (GSFC) e também no Centro de Pesquisas de Tempo e Estudos Climáticos (CPTEC). As imagens foram agrupadas em intervalos de 2 horas, para melhor observação de todo o comportamento do distúrbio analisado, totalizando 22 imagens.

Para a precipitação observada foram consultados os dados de Palmares disponíveis no CPTEC. Também foram utilizados dados de estimativas de precipitação do satélite Tropical Rainfall Measuring Mission - TRMM, com o objetivo de melhorar a distribuição espacial do evento de precipitação. $O$ algoritmo utilizado foi o 3B42 que possui resolução temporal de 3 horas e espacial de $0,25^{\circ} \times 0,25^{\circ}$. Os dados estimados têm mostrado boa correlação com os observados, e possui a vantagem da representação espacial ao contrário dos dados observados por instrumentos de medidas de chuva sobre a superfície (NÓBREGA et al., 2008a; NÓBREGA et al., 2008b).

Também foi analisado o diagrama Hovmoller do desvio meridional do vento para verificar a ocorrência de um DOL, centralizado na latitude de $10^{\circ} \mathrm{S}$, disponibilizado pelo Instituto de Astronomia, Geofísica e Ciências Ambientais da Universidade de São Paulo (IAG/USP), baseado na metodologia descrita em Cavalcanti et al. (2009). 


\section{Resultados e discussão}

\subsection{Condicionantes oceanicos e atmosféricos durante o evento}

Eventos pluviais extremos, ou de alta magnitude são aqueles considerados extraordinários dentro do ritmo climático habitual de determinada espacialidade, sendo esses os que se sobressaem tanto positivamente, pelas potencialidades que propiciam como a armazenagem d'água para atividades antrópicas, quanto negativamente, pelos prejuízos e degradação que causam pelo desencadeamento de fluxos acelerados de água com eventuais alagamentos (CHRISTOFOLETTI, 1997; GIRÃO, 2009).

Não obstante, deve-se lembrar de que são fenômenos naturais, mas, que em virtude das transformações espaciais decorrentes da degradação ambiental, bem como pela diversidade geomorfológica que atua como catalisadora dos processos de superfície, desencadeiam entradas incomuns de energia nos sistemas espaciais físicos que, de outra forma, apresentariam respostas diferentes quando da distribuição dos eventos de excepcionalidade pluviométrica (CONTI, 2001; GIRÃO, 2009).

O evento ocorrido entre 17 e 19 de junho de 2010 na Zona da Mata Sul de Pernambuco (que inclui o município de Palmares) com maior intensidade nas bacias dos rios Una e Sirinhaém, é um claro exemplo desta característica atmosférica. Os padrões normais de circulação atmosférica em grande escala determinam o mês de junho como um dos meses de maior precipitação durante a estação chuvosa na faixa litorânea de Pernambuco.

Neste episódio havia a confluência de dois sistemas atmosféricos de grande escala em atuação, acoplados à ocorrência de duas importantes anomalias sobre os oceanos. Este agrupamento de condições resultou em mudanças significativas no comportamento da precipitação. Sobre o Pacífico leste estava configurado o fenômeno La Niña com temperaturas abaixo da média, o que favorece chuvas acima da média no Nordeste do Brasil (NEB). Já sobre o Atlântico Sul, especificamente na sua porção tropical, as temperaturas estão acima da média. Segundo Nóbrega et al. (2005) e Nóbrega et al. (2010), de $98 \%$ a $95 \%$ da chuva sobre o leste do NEB tem como origem a advecção de umidade sobre o oceano Atlântico. Portanto, com temperaturas sobre o mar acima da média, tem-se maior quantidade de umidade, ou seja, o "combustível" para a chuva. 


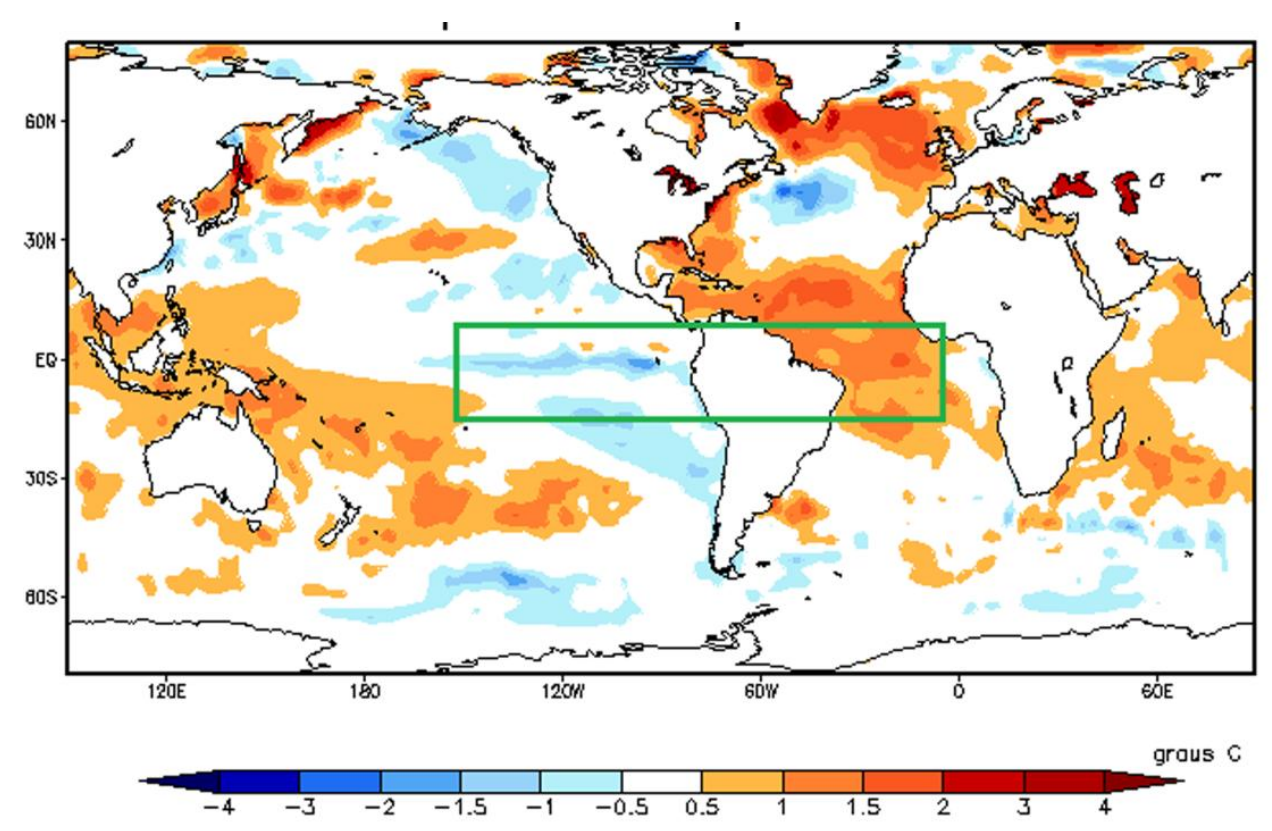

Figura 2 - Anomalias de temperatura da superfície do mar durante o mês de junho de 2010. Área realçada corresponde a região de La Ninã sobre o Oceano Pacífico e porção tropical do Atlântico Sul. Adaptado de CPTEC/INPE.

Estando as condições oceânicas propicias para ocorrência de eventos extremos, ainda é necessário que a grande escala forneça condições termodinâmicas favoráveis, como instabilidade atmosférica. Normalmente, a circulação de mesoescala sobre o leste nordestino já condiciona a instabilidade, através da circulação de brisa marítima, contudo para este episódio um intenso Distúrbio Ondulatório de Leste (DOL) configurou-se sobre a vizinhança leste do Nordeste (Figura 3). O padrão sinótico contribuiu para a formação de um DOL próximo a costa do Nordeste, favorecendo a formação de nuvens e intensificação de sistemas de precipitação desde o dia 15 de junho. O desvio zonal negativo no campo do vento meridional indica deslocamento de leste e intensificação da advecção de umidade para o continente (contorno amarelo).

A partir daqui pode-se afirmar que existiam condições oceânicas e atmosféricas que favoreceram a ocorrência de precipitação. Girão et al. (2012) observaram que a confluência de diferentes sistemas/condições atmosféricas e oceânicas que favorecem eventos pluviais tendem a intensificar os eventos, ou seja, durante eventos extremos, normalmente há a atuação de dois ou mais condicionantes para a precipitação. 


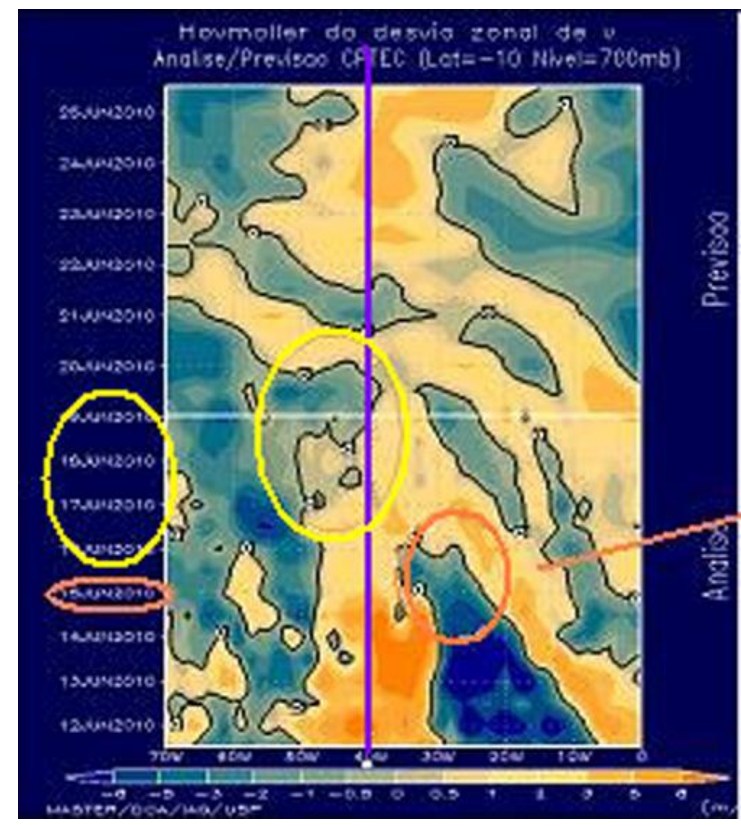

Figura 3 - Diagrama Hovmoller do desvio do vento meridional na latitude de 10S. No eixo $x$ são as longitudes e no eixo y os dias. Em torno do meridia no 40W (linha violeta) tem-se a costa nordeste da Região Nordeste. Áreas destacadas em amarelo são referentes aos dias 16 a 19 de junho de 2010, área destacada em laranja é referente ao dia 15 de junho de 2010. (Fonte: IAG/USP).

\subsection{Caracterização geral do sistema convectivo}

O sistema analisado iniciou a sua formação no dia 17 de junho de 2010 entre as 5:00 e 6:00 horas e dissipou-se no dia seguinte entre as 21:00 e 22:00 horas, num total de 41 horas de duração.

Verificou-se uma variação de tamanho durante a evolução do evento, tendo atingido um diâmetro máximo de $320 \mathrm{Km}$ no dia 18 por volta das 6:00 horas, uma dimensão considerável considerando que corresponde a aproximadamente metade do comprimento longitudinal do estado de Pernambuco. Nesse mesmo período, a temperatura de brilho no topo das nuvens variou entre $-30{ }^{\circ} \mathrm{C}$ e -40 ${ }^{\circ} \mathrm{C}$ nas bordas e $-60{ }^{\circ} \mathrm{C} \mathrm{e}-70{ }^{\circ} \mathrm{C}$ no centro (observadas nas imagens do satélite GOES, abaixo, entre as Figuras 4 a 6 ).

Disturbios ondulatórios de leste que se formam na região do Oceano Atlântico Sul deslocam-se para oeste e se intensificam a medida que se aproximam da costa leste da Região Nordeste do Brasil (NEB) (CHOU, 1990). Estes sistemas apresentam oscilações com periodicidade que varia de 3 a 9 dias, comprimento de onda que varia de 3200 a $6200 \mathrm{Km}$ e uma velocidade de propagação que varia entre 10 a $15 \mathrm{~m} / \mathrm{s}$ (DE FELICE et al., 1993; MEKONNEN et al., 2006; ALVES et al., 2008). Outra característica dos DOLs são as precipitações acima de $80 \mathrm{~mm}$ concordando que estes distúrbios são importantes, sendo este o principal sistema de grande escala potencializador para a precipitação no período outono/inverno (SILVA, 2010). 


\subsection{Ciclo de vida do evento}

\subsubsection{Gênese}

As primeiras células convectivas do evento são perceptíveis por volta das 05:00 horas do dia 17 de junho. Pela análise da figura 2, verifica-se que as bandas de nuvens, à medida que chegam a costa leste do NEB se intensificam podendo estar associada a interações do escoamento sinótico com circulações locais (TORRES, 2008; SILVA, 2010). As semanas que antecedem o evento foram bastante chuvosas, com acumulado de $260 \mathrm{~mm}$ em Recife, $235 \mathrm{~mm}$ em Palmares, por exemplo. Somente no dia 16, em Recife precipitou $116 \mathrm{~mm}$ e em Barreiros $67 \mathrm{~mm}$ (Figura 8).

\subsubsection{Maturação}

Entre as 18:00 horas (17/06) e as 11:00 horas (18/06) o DOL atingiu seu estágio maduro (Figura 3) coincidindo com valores de precipitação superiores a $100 \mathrm{~mm}$ (Figura 5). Neste estágio, na região onde o influxo dos baixos níveis fornece energia para o ambiente instável, os elementos de convecção intensa continuam a se formarem (ALVES et al., 2011).

Constata-se uma grande extensão do fluxo de massa ascendente na média troposfera e uma grande área de precipitação.

No dia 17 as cidades de Recife e Vitória de Santo Antão observaram os maiores valores de precipitação, cerca de $130 \mathrm{~mm}$ e $116 \mathrm{~mm}$, respectivamente (Figura 8). Somente entre as 15:00 horas e as 21:00 horas do dia 17, Recife observou quase $100 \mathrm{~mm}$ de precipitação e Vitória de Santo Antão observou $146 \mathrm{~mm}$ entre as 18:00 horas do dia 17 e 06:00 horas do dia seguinte. Tratam-se de fortes e intensas chuvas localizadas num curto periodo de tempo.

\subsubsection{Dissipação}

O estágio de dissipação é marcado por uma rápida mudança na estrutura do sistema. Num curto período de tempo, os elementos de intensa convecção não mais se desenvolveram, o que ocorreu por volta das 22:00 horas do dia 18 (Figura 6).

Nesta fase, o suprimento de energia para o sistema é interrompido ou modificado, não apresentando mais a estrutura em mesoescala organizada (ALVES et al., 2011).

Apesar de o sistema convectivo ter perdido a sua organização em mesoescala, o ar frio e o escoamento de umidade na camada da superfície ainda favorecem a formação de alguma nebulosidade, de maneira que pancadas de chuvas podem persistir por algumas horas. Isto é observado na Figura 10 onde os valores de precipitação no dia 19 ultrapassaram $80 \mathrm{~mm}$ de precipitação em algumas áreas de Pernambuco. 


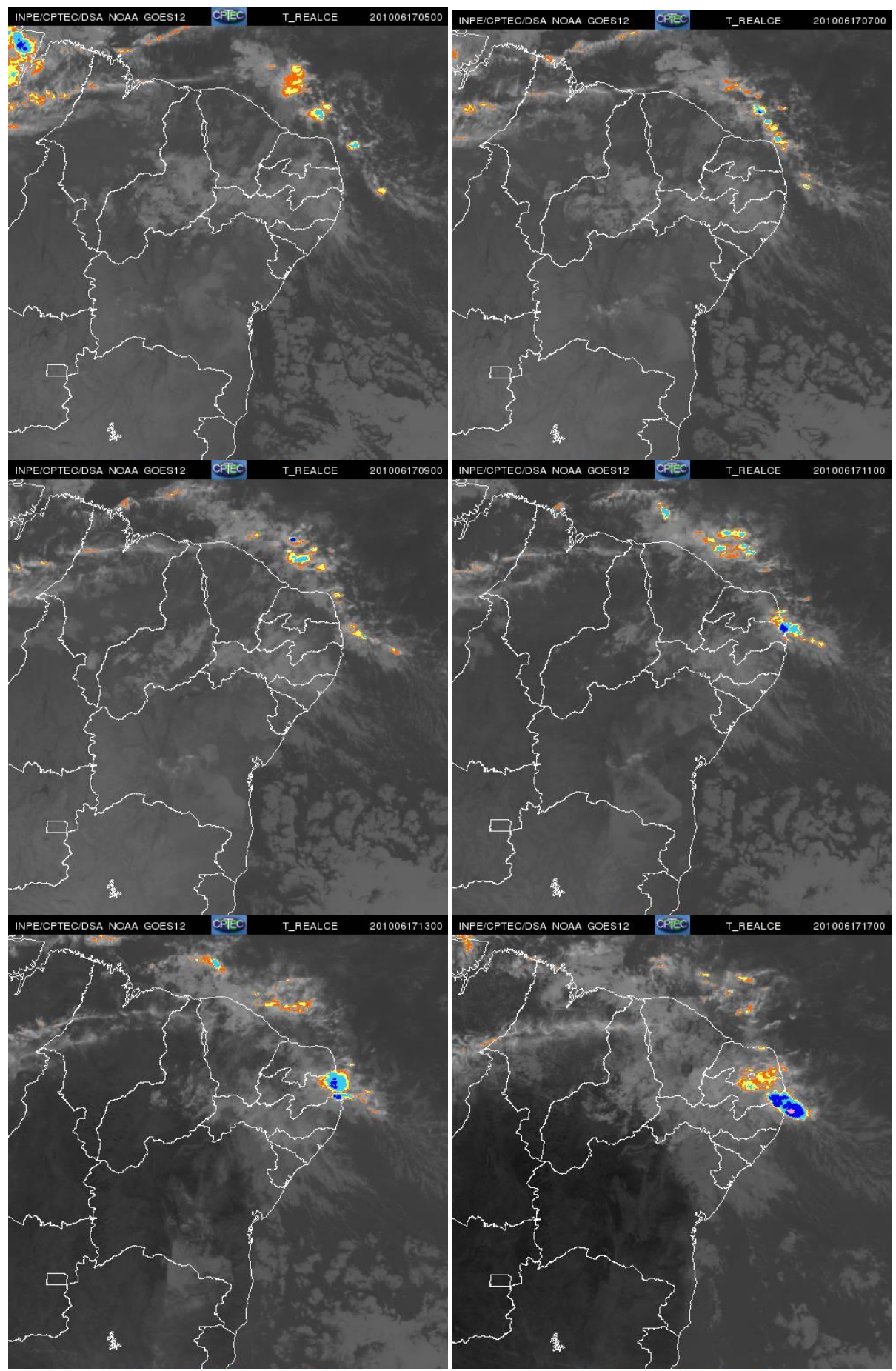

$\begin{array}{lllllll}-80 & -70 & -60 & -50 & -40 & -30 & \text { Temp. Celsius }\end{array}$

Figura 4 - Imagens do satélite GOES - 12 referentes ao início da formação do DOL. 


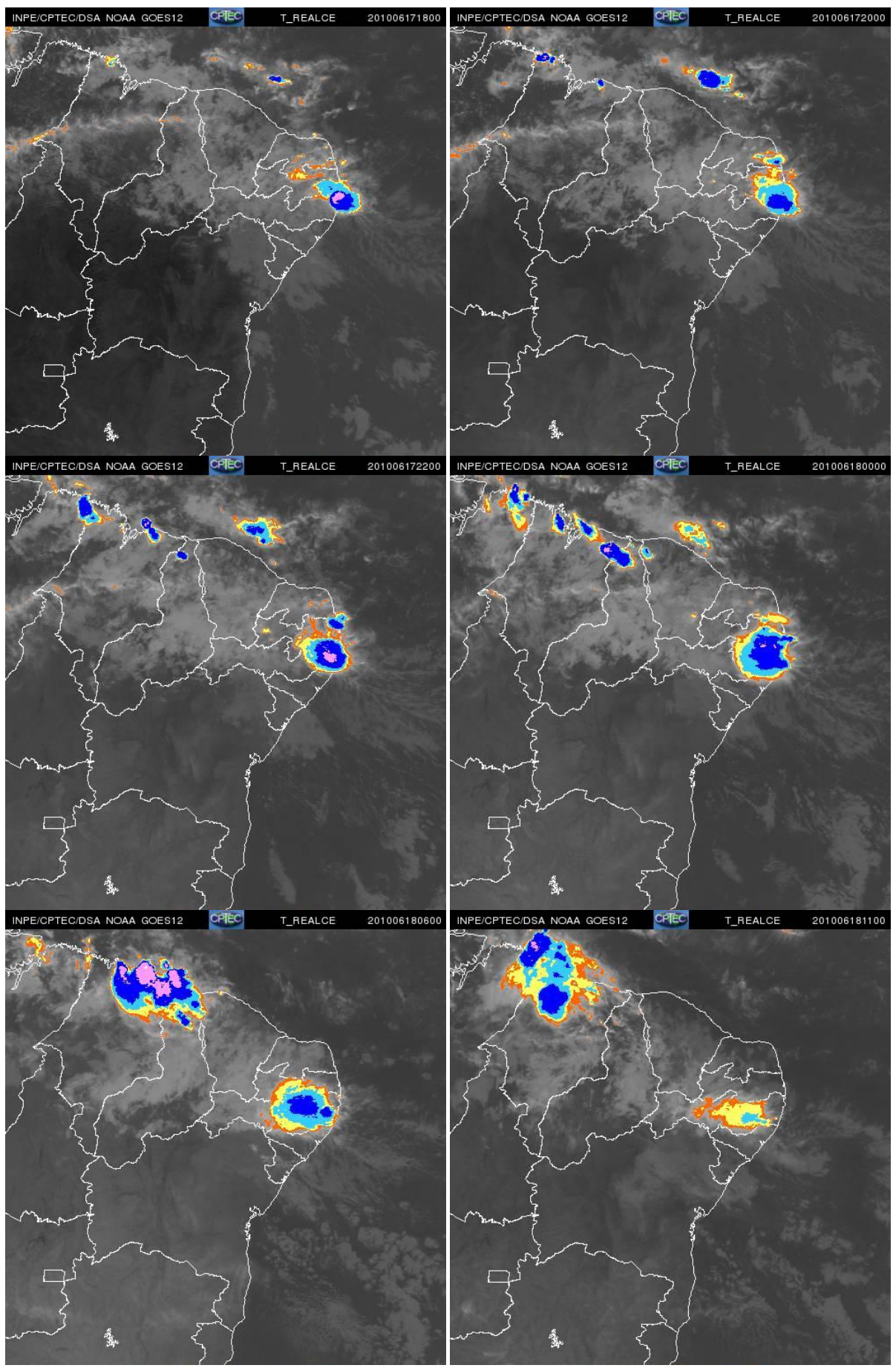

$$
\begin{array}{ccccccc}
-80 & -70 & -60 & \mathbf{- 5 0} & \mathbf{- 4 0} & -30 & \mathbf{- 3 0} \text { Temp. Celsius }
\end{array}
$$

Figura 5 - Imagens do satélite GOES - 12 referentes ao estágio de maturação do DOL.

\subsection{Efeitos do evento extremo de precipitação sobre o município de Palmares}

Os três dias de precipitação mais intensa foram dias 16, 17 e 18 de junho (Figura 7). A precipitação intensa foi se deslocando do litoral (dia 16) em 
direção ao interior onde se situam as cabeceiras dos rios (dias 17 e 18). O acumulado precipitado de 3 dias em Palmares foi de $65 \mathrm{~mm}$, mas em outras áreas supera $250 \mathrm{~mm}$. De fato, as precipitações ocorridas em Palmares foram intensas, no entanto, as precipitações ocorridas a montante, nas cabeceiras das bacias, foram excepcionais (Figura 7). Esse fato foi preponderante para as cheias devastadoras ocorridas no município de Palmares no dia 18 e 19 de junho.

Pode-se observar na Figura 8 que a precipitação foi mais intensa na latitude $7,75^{\circ}$ S e $8,75^{\circ}$ S no dia $18 / 06$ com valores superiores a $100 \mathrm{~mm}$ e $70 \mathrm{~mm}$, respectivamente. De acordo com Silva (2010), os eventos de precipitação condicionados por DOLs que atingem o NEB são mais frequentes entre as latitudes de $50 \mathrm{~S}$ e $7,5^{\circ} \mathrm{S}$.

A precipitação foi acima da média com deslocamento de montante para jusante e concentração em três dias. Considerando que as semanas que antecederam o evento foram bastante chuvosas, os açudes que se distribuem pela bacia, e que normalmente retém parcela importante da precipitação evitando escoamento superficial, já se encontravam saturados. Esse fato adicionado a solos rasos com baixa capacidade de armazenamento característicos da área, resultaram em coeficientes de escoamento superficial acima da média (GIRÃO et al., 2012; JúNIOR et al., 2010).

Outro fator a ser considerado para justificar a devastação ocorrida em Palmares é a elevada declividade da bacia. Como pode ser observado na Figura 1, a nascente do rio Una ocorre acima de $820 \mathrm{~m}$ de altitude e corre ao longo de, aproximadamente, $255 \mathrm{~km}$ até desaguar no oceano Atlântico, determinando o alto gradiente do rio. O rio Una percorre apenas $120 \mathrm{~km}$ entre sua nascente e a cidade de palmares, que se encontra a $125 \mathrm{~m}$ de altitude. Esta diferença de altitude para relativa pequena distância explica a elevada energia cinética do rio quando atinge a cidade.

Por fim, a ocupação das planícies de inundação dos rios por parte da população de Palmares potencia a destruição causada por um evento já de si devastador. Em Palmares, bairros inteiros foram construídos dentro do rio (Figura 10). Apesar do perigo que isso representa, a população se habituou a conviver com as cheias do rio, e evento após evento, ela volta a ocupar a calha maior do rio, explicando os muitos estragos recorrentemente registrados. 
Revista Brasileira de Climatologia

ISSN: 1980-055x (Impressa) 2237-8642 (Eletrônica)

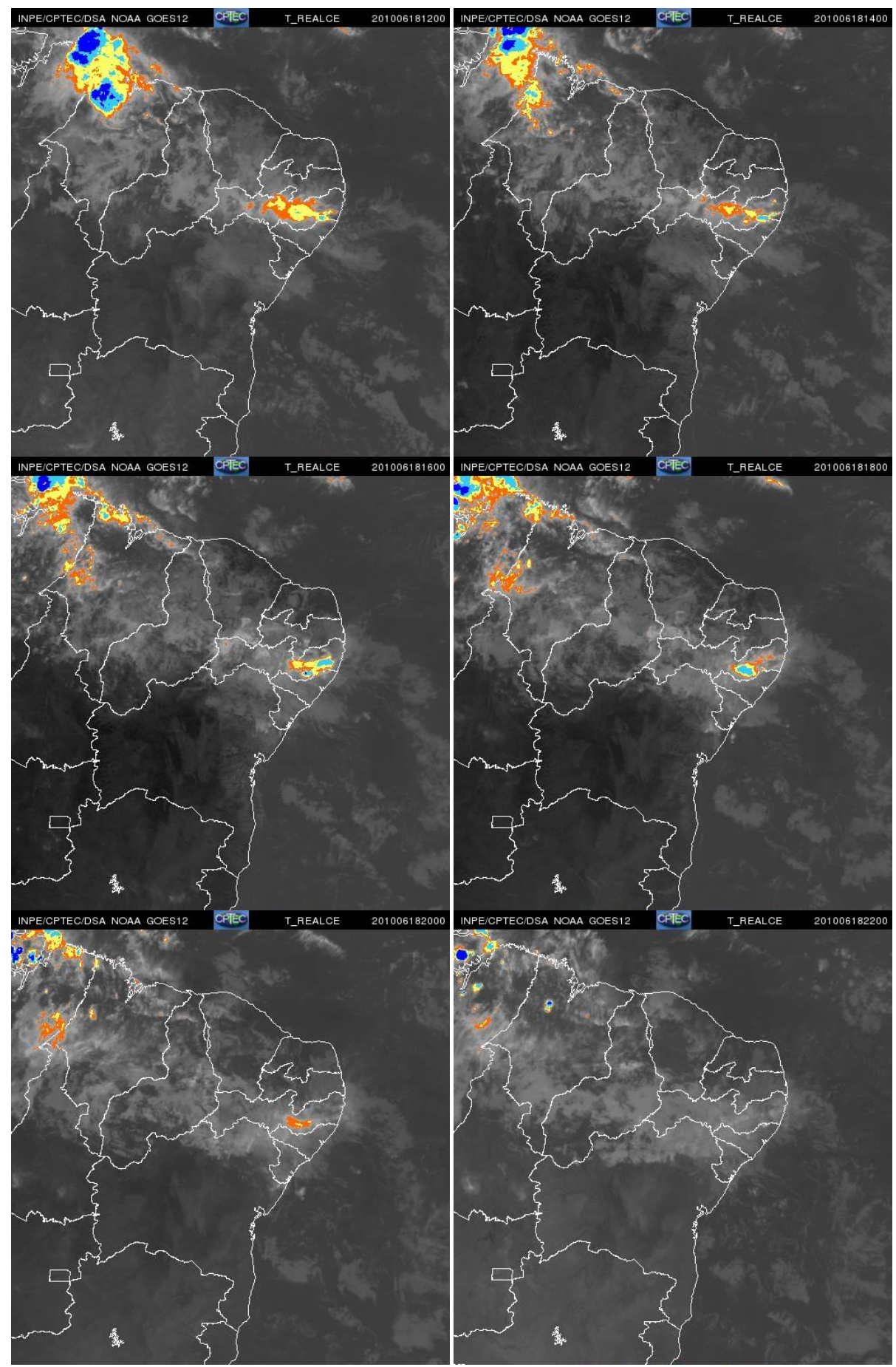

$\begin{array}{lllllll}-80 & -70 & -60 & -50 & -40 & -30 & \text { Temp. Celsius }\end{array}$

Figura 6 - Imagens do satélite GOES - 12 referentes ao estágio de dissipação do DOL. 

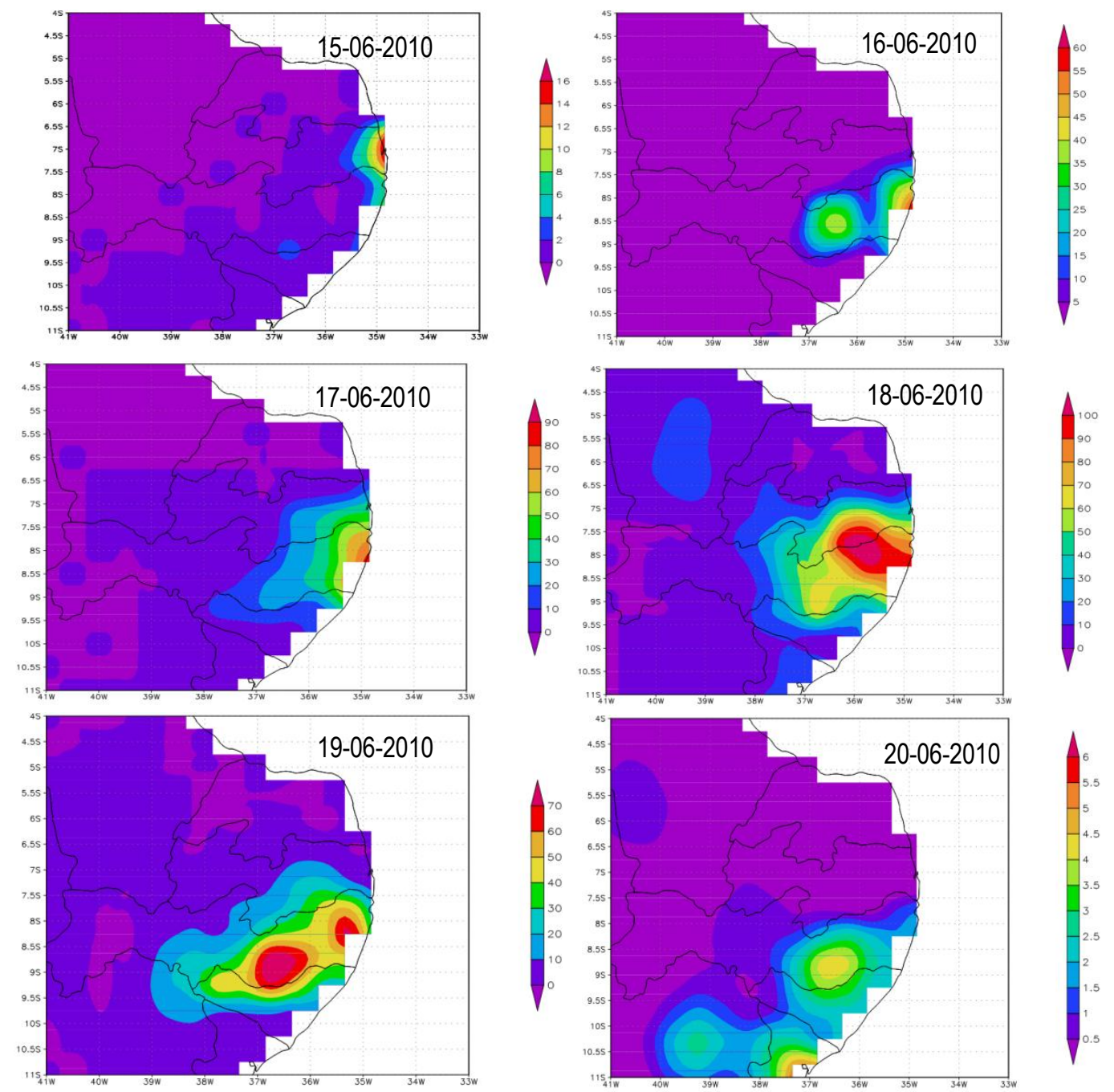

Figura 7 - Estimativa de precipitação pelo satélite TRMM acumulada para os dias 15 a 20 de junho de 2010 ( $\mathrm{mm})$. 

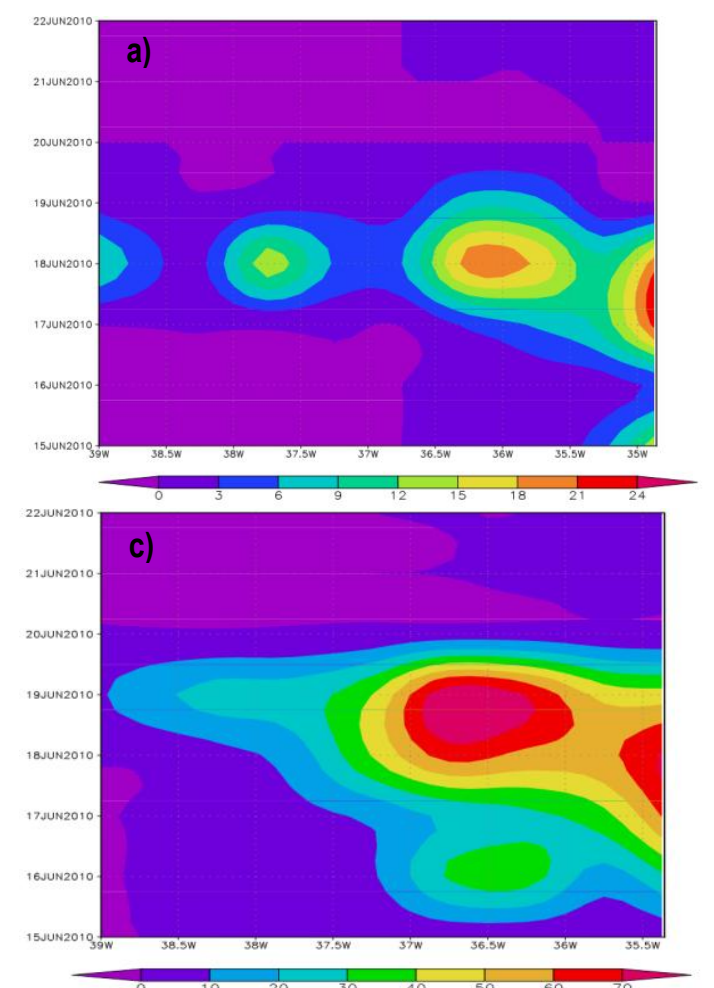
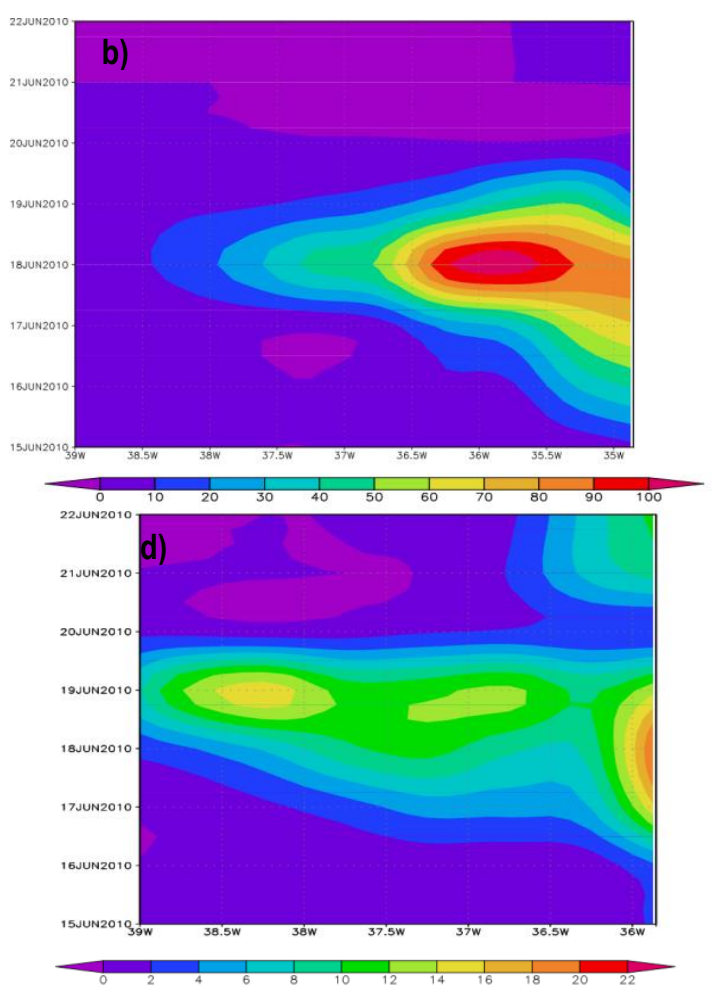

Figura 8 - Corte longitudinal de precipitação estimada através do satélite TRMM acumulada para os dias 15 a 20 de junho de $2010(\mathrm{~mm})$ - a) latitude $6,75^{\circ}$; b) latitude $7,75^{\circ}$; c) latitude $8,75^{\circ} \mathrm{e}$; d) latitude $9,75^{\circ}$.

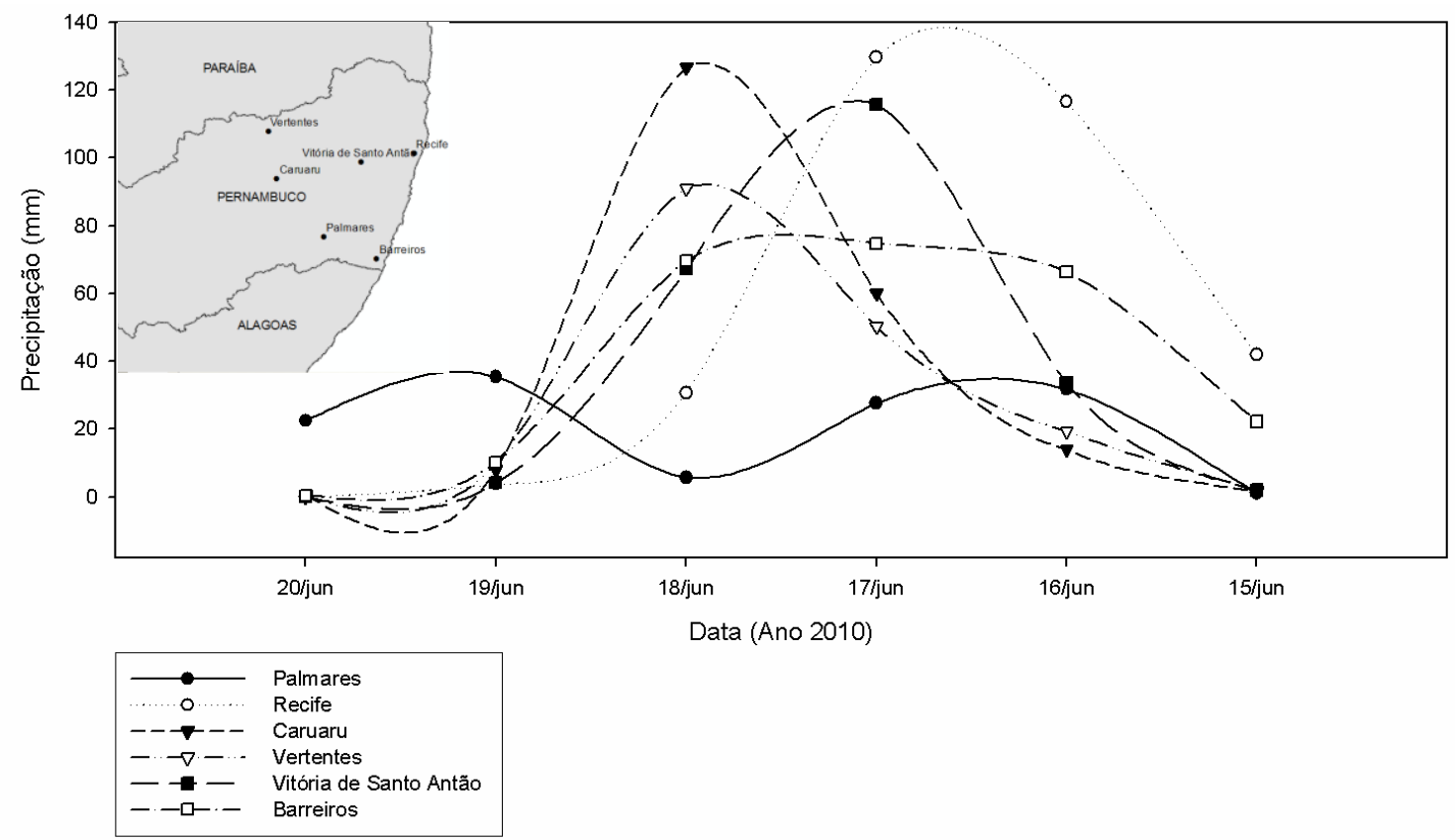


Figura 9 - Valores de precipitação $(\mathrm{mm})$ obtidos a partir das PCD (CPTEC) de Palmares, Recife, Caruaru, Vertentes, Vitória de Santo Antão e Barreiros entre os dias 15 e 20 de junho de 2010.

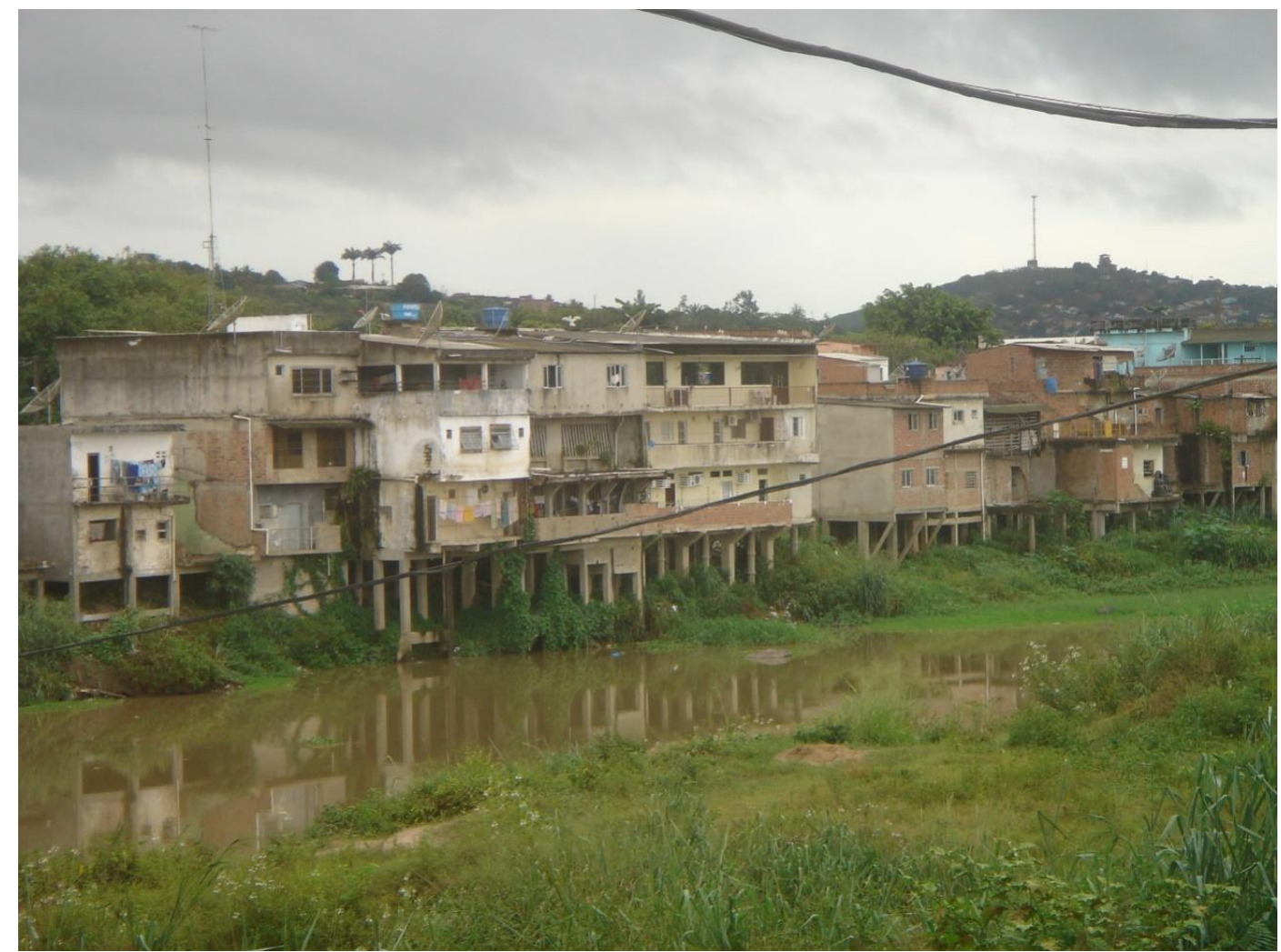

Figura 10 - Foto de casas construídas em cima do rio Una na cidade de Palmares. (Abril, 2011).

\section{CONCLUSÕES}

A ocorrência de um Distúrbio Ondulatório de Leste acoplado a condições de temperaturas de superfície do mar anômalas modulou a intensificação de um sistema convectivo de mesoescala, que produziu precipitações acima dos padrões normais durante 3 dias.

O sistema de DOL pelo seu tempo de sua duração e pelos valores de precipitação superiores a $100 \mathrm{~mm}$ indicam que esse distúrbio é um sistema importante para ocorrência de chuva na costa leste do NEB.

A utilização de imagens do satélite GOES-12 devidamente realçada para destaque da temperatura das nuvens, permitiu o monitoramento do evento de precipitação de forma eficiente. A sua resolução temporal de 15 minutos possibilita o acompanhamento da atuação de sistemas de curta duração e de mudanças rápidas.

A leitura dos dados meteorológicos e das estimativas de precipitação extraídas do satélite TRMM possibilitam análise mais detalhada, sobretudo temporoespacial, do comportamento do DOL e de suas consequências. 
Quanto aos impactos ocorridos no município de Palmares em junho de 2010, uma série de fatores foram responsáveis e não apenas o evento em si. As elevadas precipitações ocorridas nas semanas anteriores ao evento e sua concentração em três dias principalmente nas cabeceiras, as características do solo e do relevo e a ocupação das planícies de inundação pela população agravaram para proporções catastróficas um evento já em si muito intenso.

\section{REFERÊNCIAS}

ALVES, M. A. S.; MARQUES, R. F. C.; OYAMA, M. D. Deteç̧ão de DOL a partir de filtros temporais. In: Congresso Brasileiro de Meteorologia, 2008, São Paulo. Anais... São Paulo: CBMET, 2008.

ALVES, K.M.A.S; NOBRÉGA, R.S.; CAVALCANTI, L.C.S.; MELO, H.L.C.F. Estudo de caso de aspectos dinâmicos de um Complexo Convectivo de Mesoescala (CCM) em Pernambuco através de sensores remotos. In: XV Simpósio Brasileiro de Sensoriamento Remoto, 2011, Curitiba. Anais... Curitiba: SBSR, 2011.

BARBOSA, R.L. Interação das perturbações convectivas iniciadas na costa Norte do Brasil com Distúrbios Ondulatórios de Leste. São José dos Campos. 2005. 81 p. Dissertação (Mestrado em Meteorologia) - Instituto Nacional de Pesquisas Espaciais - INPE.

CAVALCANTI, I. F.; FERREIRA, NL. J.; DA SILVA, M. G. A.; SILVA DIAS, M. A. Tempo e Clima no Brasil, São Paulo: Editora Oficina de Textos, 463 p., 2009.

COOK, K.H.; PATRICOLA, C.M.; VIZI, E.K. Easterly Waves in the tropical Atlântic: climatology and variability. Monthly Weather Review. 2004.

CHOU, S.C. Análise de Distúrbios Ondulatórios de Leste sobre o oceano Atlântico Equatorial Sul. 1990. 153 p. (INPE - 5222 - TDL / 437). Dissertação (Mestrado em Meteorologia) - Instituto Nacional de Pesquisas Espaciais, São José dos Campos. Acesso em: 26 jun. 2011. Disponível em: http://urlib.net/sid.inpe.br/MTC-m12@80/200

5/08.18.12.54.

CONTI, J. B. Geografia e Climatologia. Geousp, n.9, p. 91-95, 2001.

COOK, K.H.; PATRICOLA, C.M.; VIZI, E.K. Easterly Waves in the tropical Atlantic: climatology and variability. Monthly Weather Review. 2004.

COUTINHO, E.C.C.; FISCH, G. Distúrbios Ondulatórios de Leste (DOLs) na região do centro de lançamento de Alcântara-MA. Revista Brasileira de Meteorologia, v.22, n.2, 193-203, 2007.

CHRISTOFOLETTI, A. Complexidade e auto-organização aplicadas em estudos sobre paisagens morfológicas fluviais. In: SIMPÓSIO BRASILEIRO DE GEOGRAFIA FÍSICA APLICADA, 7., 1997, Curitiba. Anais... Paraná: Universidade Federal do Paraná, 1997. p. 9-19.

DE FELICE, P.; VILTARD, A., OUBUIH, J. A synoptic scale wave 6-9 day period in the Atlantic Tropical Troposphere During Summer 1981. Monthly Weathear Review, v. 121, p. 1291-81, 1993. 
JÚNIOR, C.R.F.; PEDROSA, V.A.; DE SOUZA, V.C.B. Reflexões sobre a cheia de junho de 2010 nas bacias do Rio Mundaú e Paraíba. In: Simpósio Regional Brasileiro de Recursos Hídricos, 2010, Fortaleza. Anais... Fortaleza: SRBRH, 2010.

GIRÃO, O. Mudanças Climáticas Globais: Impactos sobre o espaço nordestino - o aumento dos eventos pluviais extremos. Revista de Geografia (Recife), v. 26, p. 216-255, 2009.

GIRÃO, O.; CORREA, A. C. B.; NÓBREGA, R. S.; DUARTE, C. o papel do clima nos estudos de prevenção e diagnóstico de riscos geomorfológicos em bacias hidrográficas na zona da mata sul. No prelo. 2012

MACHADO, L.A.T.; GUEDES, R.L.; ALVES; M.A.S. Características estruturais de sistemas convectivos e forçantes da convecção na América do Sul observados por satélites. Climanálise 10 anos, CPTEC/INPE, Cachoeira Paulista. 1996, p. 110-122.

MEKONNEN, A.; TRORNCROFT, C. D.; AIYYER, A. R. Analysis of Convection and Its Association white African Easterly Waves. Journal of Climate. V. 19, p. 5405-5421, 2006.

MOTA, G.V. Estudo observacional de distúrbios ondulatórios de leste no nordeste brasileiro. 1997. 93p. Dissertação (Mestrado em Meteorologia) - Instituto Astronômico e Geofísico, Universidade São de Paulo, São Paulo, 1997.

NÓBREGA, R. S.; SILVA, D. F. da; ARAÚJO, L. E. de; SOUZA, F. A. S. de. Detecção do início, fim e duração da estação chuvosa na Paraíba através da técnica dos quantis. In: Simpósio Internacional de Climatologia, 2005, Fortaleza. Anais... Fortaleza: SIC, 2005.

NÓBREGA, R.S.; SOUZA, Ê. P. de; GALVÍNCIO, J.D.. Análise da estimativa de precipitação do TRMM em uma sub-bacia da Amazônia Ocidental. Revista de Geografia (Recife), v.25, p.5-19, 2008 a.

NÓBREGA, R.S.; SOUZA, Ê.P. de; SOUZA, F.A.S. de. Análise da utilização de dados do satélite TRMM em um modelo hidrológico semidistribuído na bacia do rio Jamari (RO). Revista Brasileira de Geografia Física, v.1, p.47-61, 2008b.

NÓBREGA, R. S.; SOUZA, Ê. P. de ; SOUZA, F. A. S. de . The impacts of changes in land cover on water resources in the western Amazon. Journal of Environmental Hydrology, v. 18, p. 1-12, 2010.

SILVA, V.P. Distúrbios Ondulatórios de Leste: estudo de casos que afetaram a costa leste do NEB. 2010. 77p. Dissertação (Mestrado em Meteorologia) Centro de Tecnologia e Recursos Naturais Unidade Acadêmica de Ciências Atmosféricas, Universidade Federal de Campina Grande, Campina Grande, 2010.

TORRES, R. Analise e Modelagem dos Distúrbios Ondulatórios de Leste que atuam na Costa Leste do Nordeste do Brasil. set. 2008. (INPE-15459-TDI/1421). Dissertação (Mestrado em Meteorologia) - Instituto Nacional de Pesquisas Espaciais, São José dos Campos. Disponível em: http://urlib.net/sid.inpe.br/mtcm18@80/2008/08.20.23.59. Acesso em: 26 jun. 2011.

ZEHNDER, J.A.; POWELL, D.M.; ROPP, D.L. The Interaction of Easterly Waves, Orography, and the Intertropical Convergence Zone in the Genesis of Eastern Pacific Tropical Cyclones. Monthly Weather Review, v.127, p.1566-1585. 1999. 